\title{
Design of binary long-period fiber grating filters by the inverse-scattering method with genetic algorithm optimization
}

\author{
Gia-Wei Chern and Lon A. Wang \\ Department of Electrical Engineering and Institute of Electro-Optical Engineering, National Taiwan University, \\ Taipei, Taiwan, China
}

Received February 23, 2001; revised manuscript received August 2, 2001; accepted August 30, 2001

\begin{abstract}
An approach is presented to the design of binary long-period fiber grating (LPFG) filters based on the Gel'fand-Levitan-Marchenko (GLM) inverse-scattering method and genetic algorithm optimization. The nonuniform coupling strength of the binary grating can be realized by varying the local duty ratio. A coupledmode theory combined with the Poisson sum formula for treating the binary index perturbation is developed for the application of the GLM synthesis method. Since the coupled-mode theory, which smears out the discrete coupling nature, can be regarded only as an approximation to the modeling of a binary LPFG, we use instead the transfer-matrix model to analyze the coupling behavior of a nonuniform binary LPFG. Based on the synthesized grating patterns from the GLM method, a real-coded genetic algorithm with the transfermatrix model is used to compensate for the discrepancies resulting from use of the coupled-mode theory and to optimize the design. We exemplify the above procedure by designing a flatband LPFG filter and a highvisibility all-fiber Mach-Zehnder filter. (C) 2002 Optical Society of America
\end{abstract}

OCIS codes: $050.2770,060.2310,060.2340$.

\section{INTRODUCTION}

A long-period fiber grating (LPFG) provides transmissiontype loss filters by coupling optical power from the fundamental core mode to the phase-matched cladding modes. $^{1,2}$ The transmission spectrum of an LPFG consists of many dips that can be attributed to light couplings from the core mode to various cladding modes. Such all-fiber optical filters play an important role in optical communication systems. One important application of an LPFG is as a gain equalizer for an erbium-doped fiber amplifier (EDFA). ${ }^{3}$ The transmission dip of a uniform LPFG corresponding to a specific cladding mode resonance has a sinclike profile with appreciable sidelobes. To construct a loss filter with an inverted excess gain spectrum of the EDFA, two uniform LPFGs with different loss depths and center wavelengths are concatenated to realize the desired filter response in Ref. 3 . When a phase shift is placed in the middle point of a uniform LPFG and the length of the phase shift is properly adjusted, the original loss dip corresponding to coupling from the core mode to a specific cladding mode splits into two dips, with relative peak losses controlled by the amount of the inserted phase shift. Such an LPFG can also be used as a gain-flattening filter for an EDFA. ${ }^{4}$ Recently, Bae et al. proposed a multiport lattice filter model for synthesizing piecewise-uniform LPFGs and applied it to the gain equalization of an EDFA. ${ }^{5}$ In addition to gain equalization, other optical communication applications may require special filters with arbitrary amplitude and phase responses. It is the goal of this paper to propose a general solution to the synthesis problem of a nonuniform binary LPFG.

Many methods have been proposed to fabricate a complex-patterned fiber Bragg grating (FBG) whose period is submicrometer; e.g., by the moving fiber-scanning beam technique. ${ }^{6}$ Conversely, since the grating period of an LPFG is tens to hundreds of micrometers, the linewidth of the mask for fabricating a complex nonuniform LPFG will be larger. Since most LPFGs are fabricated by using amplitude masks, the resultant LPFG assumes a binarylike index variation. Those regions exposed to ultraviolet irradiation will thus be termed as regions 1, in which the core index is slightly increased as a result of photosensitivity of the germanosilicate core. The other regions are of a conventional fiber structure and will be referred to as regions 0 . The transfer-matrix method for modeling the photoinduced binary LPFG based on a mode-matching technique and perturbation expansions have recently been developed by the authors in Ref. 7 . Nonuniformity of the coupling strength of a binarylike waveguide grating, e.g., a grating-assisted codirectional coupler (GACC), can be realized by varying the local duty ratios. ${ }^{8,9}$ We shall use this principle to propose a design procedure for a nonuniform binary LPFG with the desired spectral response in the following and use the transfermatrix model to analyze the grating response. Unlike the fabrication of an FBG, this method has the advantage that no scanning steps or positioning facilities are needed for the fabrication of the complex-patterned LPFG. Once the variation of the duty ratio and the local period is determined for the binary LPFG with the desired spectrum, one has only to fabricate the amplitude mask with the designed pattern of $0 \mathrm{~s}$ and $1 \mathrm{~s}$. By direct exposure through the amplitude mask, the designed pattern can easily be transferred to a fiber. Our procedure for the design of a binary LPFG is as follows. A coupled-mode theory based 
on the Poisson sum formula for treating the nonuniform binary index perturbation is developed for the analysis of a nonuniform binary grating. ${ }^{10}$ Then, by applying the well-developed Gel'fand-Levitan-Marchenko (GLM) inverse-scattering method, ${ }^{11}$ one can determine the desired variation of duty ratio and local period. The GLM inverse-scattering method has been successfully applied to the filter design of an FBG (e.g., Poladian ${ }^{12}$ and Feced et $\left.a l .{ }^{13}\right)$. However, a discrepancy from the desired filter response arises when the transmission spectrum of the LPFG is calculated by employing the transfer-matrix model with grating parameters derived from the GLM method. This is because the GLM method is based on the coupled-mode theory, which smears out the discrete coupling characteristic of a binary grating, in which mode couplings are assumed to take place only at the heterointerfaces of regions 0 and 1 . In addition, we define a dimensionless parameter that can be used to characterize the self-coupling effect for any GACC. And it is found that the larger the parameter, the greater the corresponding discrepancy from the desired spectrum. A genetic algorithm is then used to compensate for the effect of selfcouplings and to optimize the designed grating pattern.

The remainder of this paper is organized as follows. In Section 2, we first develop a coupled-mode theory based on the Poisson sum formula for analysis and synthesis of nonuniform binary grating filters. The index perturbation of a binary grating is transformed into a quasiFourier series whose expansion coefficients act as the tapered coupling constants. Coupled-mode equations are then used to analyze the grating responses. The discussion follows closely our previous work on the design of GACC filters. ${ }^{10}$ Section 3 is devoted to the transfermatrix model for binary LPFGs. We reformulate the method for connection with the result of coupled-mode theory. An effective local coupling coefficient is derived by using the matrix method and shows discrepancies from the coupling coefficient derived from the quasi-Fourier series in Section 2. It is shown that the discrepancy is mainly due to the nonzero difference between the selfcoupling constants of the two resonantly coupled modes. In Section 4, we apply the method to design a flatband grating filter. A genetic algorithm is used to compensate for the discrepancies and to optimize the derived grating pattern from the GLM method. By symmetrically cascading a pair of the above designed flatband LPFG filters, we obtain an all-fiber Mach-Zehnder (MZ) filter. We calculate the filter response by taking into account the waveguide dispersion, and the spectrum of the MZ filter shows a high fringe visibility. Finally, we make our conclusion in Section 5.

\section{COUPLED-MODE THEORY FOR NONUNIFORM BINARY WAVEGUIDE GRATINGS}

In this section, we will develop the coupled-mode theory for nonuniform binary waveguide gratings. The index perturbations of a binary grating take only two values for a specific transverse part, corresponding to regions 1 and 0 . Nonuniformity of the grating is due to the varying local duty ratios and grating periods. We first use the Pois- son sum formula to derive the quasi-Fourier-series expansion of the index perturbation for such nonuniform binary gratings. The expansion coefficients in the case of nonuniformity are also functions of the longitudinal distance. Then conventional coupled-mode theory is used to describe mode couplings of the grating. The zeroth-order expansion coefficient function contributes to selfcouplings of the interacting guided modes, which will modify the corresponding propagation constants, while the phase-matched $m$ th-order term is responsible for the energy transfer between the resonantly coupled modes. The following discussion parallels closely the analysis of a nonuniform GACC in Ref. 10. A schematic diagram of the index perturbation of a nonuniform binary LPFG is shown in Fig. 1. Here we have chosen the $z$ axis as the longitudinal direction of the grating. We take the transverse guiding structure of region 0 as the unperturbed one and let $\Delta n_{g}^{2}(x, y)$ be the index perturbation of region 1. The index perturbation of the photoinduced binary LPFG is $\Delta n_{g}^{2} \cong 2 n_{\text {co }} \Delta n_{\text {UV }}$ in the fiber core and zero elsewhere, where $\Delta n_{\mathrm{UV}}$ is the photoinduced index change. The center position of the $n$th region 1 is designated as $z_{n}$, and the corresponding width as $w_{n}$. Then we can express the index perturbation as follows:

$$
\Delta n^{2}(x, y, z)=\sum_{n=1}^{N} \Delta n_{g}^{2}(x, y) u\left(\frac{z-z_{n}}{w_{n}}\right)
$$

where $u(z)$ is the unit square function, which is unity when $|z| \leqslant 0.5$ and zero elsewhere. The summation can be formally extended from $-\infty$ to $\infty$ if one assumes that $w_{n}=0$ for $n<1$ or $n>N$. The center positions and widths of region 1 are regarded as sampled values of two continuous functions; i.e., $z_{n}=z(n)$ and $w_{n}=w(n)$. We next transform the above summation into a quasiFourier-series expansion by invoking the Poisson sum formula. This formula was originally adopted by Ishimaru in analyzing the array factor of a nonuniformly spaced antenna array. ${ }^{14}$ The Poisson formula is

$$
\sum_{n=-\infty}^{\infty} f(n)=\sum_{m=-\infty}^{\infty} \int_{-\infty}^{\infty} f(n) \exp (i 2 m \pi n) \mathrm{d} n
$$
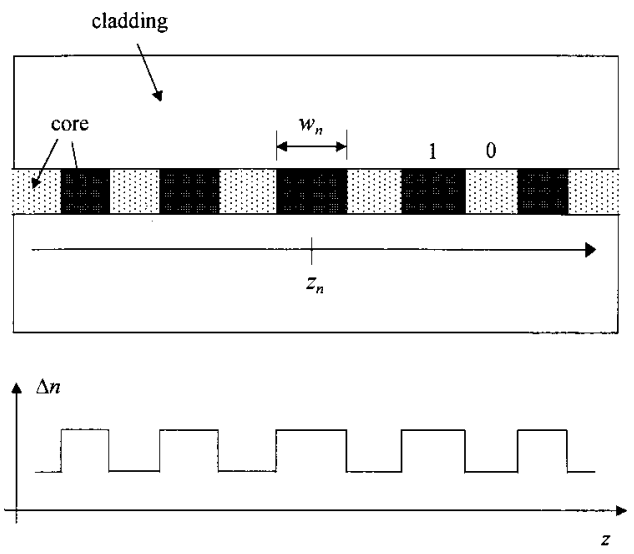

Fig. 1. Schematic diagram of a binary LPFG. The shadowed fiber core corresponds to region 1 , while the dotted one is region 0 . Also shown is the index perturbation along the longitudinal distance. 
where $f(n)$ is a continuous function of $n$. The transformed index perturbation becomes

$$
\begin{aligned}
\Delta n^{2}(x, y, z)= & \Delta n_{g}^{2}(x, y) \sum_{m=-\infty}^{\infty} \int_{-\infty}^{\infty} u\left[\frac{z-z(n)}{w(n)}\right] \\
& \times \exp (i 2 m \pi n) \mathrm{d} n .
\end{aligned}
$$

Note that the summation over $m$ is basically a generalization of the conventional Fourier-series expansion. The expansion coefficients in Eq. (3) are also functions of the longitudinal distance. Since $z_{n}$ always increases with $n$, the position function $z(n)$ is a monotonically increasing function and is thus invertible. Let the inverse function be $n(z)$, which is called the source-number function. ${ }^{14}$ Since the grating is assumed to be almost periodic, this function takes the following form:

$$
n=n(z)=\frac{z}{\bar{\Lambda}}+\nu(z),
$$

where $\bar{\Lambda}$ is a reference period and $\nu(z)$ is a slowly varying function of $z$. Additionally, $\nu(z) \ll 1$ for an almostperiodic grating. The derivative of the source-number function, $\mathrm{d} n / \mathrm{d} z \cong \Delta n / \Delta z_{n+1, n}=1 / \Lambda_{n}$, has the physical meaning of the inverse of the local period; thus we define the local period function $\Lambda(z)$ as

$$
\frac{1}{\Lambda(z)} \equiv \frac{\mathrm{d} n}{\mathrm{~d} z}=\frac{1}{\bar{\Lambda}}+\frac{\mathrm{d} \nu}{\mathrm{d} z} .
$$

We also define the local duty ratio $\mu(z)$ as

$$
\mu(z) \equiv \frac{w(z)}{\Lambda(z)} .
$$

By changing integration variables from $n$ to $z$ in the quasi-Fourier-series expansion (3) and using the slowly varying properties of the local parameters, we obtain the following result (the details of the derivation can be found in Ref. 10):

$$
\begin{aligned}
\Delta n^{2}(x, y, z)= & \sum_{m=-\infty}^{\infty} \Delta \hat{n}_{m}^{2}(x, y ; z) \\
& \times \exp \left[i m \int_{0}^{z} \frac{2 \pi}{\Lambda\left(z^{\prime}\right)} \mathrm{d} z^{\prime}\right],
\end{aligned}
$$

with the expansion coefficient functions

$$
\Delta \hat{n}_{m}^{2}(x, y ; z)=\left\{\begin{array}{ll}
\Delta n_{g}^{2}(x, y) \mu(z), & m=0 \\
\Delta n_{g}^{2}(x, y) \frac{\sin [\mu(z) m \pi]}{m \pi}, & m \neq 0
\end{array} .\right.
$$

Note that in Eq. (7), the quasi-Fourier series is expanded by use of the local grating period $\Lambda(z)$ in integral form but not the constant reference period $\bar{\Lambda}$. Now we apply the above results to the codirectional couplings of guided modes in binary gratings, e.g., GACCs or LPFGs. We consider the case of resonant two-mode couplings and let the two nearly phase-matched modes be modes 1 and 2 . For practicality, the two resonantly coupled modes of a binary LPFG are chosen to be the fundamental core mode
$\left(\mathrm{LP}_{01}\right)$ and the cladding mode of order $\nu\left(\mathrm{LP}_{0 \nu}\right)$. By neglecting the backward-propagating modes, we can write the total electric field as

$$
\mathbf{E}(\mathbf{r})=\sum_{j=1,2} A_{j}(z) \mathbf{e}_{j}(x, y)=\sum_{j=1,2} a_{j}(z) \exp \left(i \beta_{j} z\right) \mathbf{e}_{j}(x, y),
$$

where $\left(\mathbf{e}_{j}, \mathbf{h}_{j}\right)$ are the mode fields, which are assumed to be mutually orthogonal and normalized according to

$$
\frac{1}{2} \int_{A_{\text {со }}}\left[\mathbf{e}_{j} \times \mathbf{h}_{k}\right] \cdot \mathbf{z} \mathrm{d} A=\delta_{j k} \quad(j, k=1,2) .
$$

Here $\delta_{j k}$ is the Kronecker delta symbol. $\quad \beta_{j}$ is the propagation constant of mode $j$, and $a_{j}(z)$ is the corresponding slowly varying mode amplitude. Without loss of generality, we shall now take the resonance grating order to be $m=1$. By substituting Eqs. (7) and (8) into the coupledmode equation, ${ }^{2,15}$ we obtain the evolution of the mode amplitudes:

$$
\begin{aligned}
\frac{\mathrm{d} a_{1}}{\mathrm{~d} z}= & i \Delta \beta_{1}(z) a_{1}+i K(z) a_{2} \\
& \quad \times \exp \left\{-i \int_{0}^{z}\left[\beta_{1}-\beta_{2}-\frac{2 \pi}{\Lambda(z)}\right] \mathrm{d} z\right\}, \\
\frac{\mathrm{d} a_{2}}{\mathrm{~d} z}= & i \Delta \beta_{2}(z) a_{2}+i K^{*}(z) a_{1} \\
& \quad \times \exp \left\{i \int_{0}^{z}\left[\beta_{1}-\beta_{2}-\frac{2 \pi}{\Lambda(z)}\right] \mathrm{d} z\right\},
\end{aligned}
$$

where $\Delta \beta_{j}(z)$ is the local correction to the propagation constant of mode $j$, given by

$$
\Delta \beta_{j}(z) \equiv \kappa_{j j} \mu(z) \quad(j=1,2),
$$

and the cross-coupling coefficient is

$$
K(z) \equiv \kappa_{12} \frac{\sin [\mu(z) \pi]}{\pi} .
$$

Here we introduce the following coupling constant:

$$
\kappa_{j k}=\frac{\omega \varepsilon_{0}}{4} \int_{A_{\infty}} \Delta n_{g}^{2}\left(\mathbf{e}_{t j}^{*} \cdot \mathbf{e}_{t k}+\frac{\bar{n}^{2}}{n^{2}} \mathbf{e}_{z j}^{*} \mathbf{e}_{z k}\right) \mathrm{d} A,
$$

where $\bar{n}^{2}(x, y)$ is the transverse index profile of region 0 , $n^{2}=\bar{n}^{2}+\Delta n_{g}^{2}$ is that of region 1 , and the subscripts $t$ and $z$ indicate the transverse and longitudinal parts of the electric fields, respectively. With the mode amplitude transformations

$$
\hat{a}_{j}(z)=a_{j}(z) \exp \left[-i \int_{0}^{z} \Delta \beta_{j}\left(z^{\prime}\right) \mathrm{d} z^{\prime}\right] \quad(j=1,2),
$$

the coupled-mode equations (11) become

$$
\begin{aligned}
& \frac{\mathrm{d} \hat{a}_{1}}{\mathrm{~d} z}=i K(z) \hat{a}_{2} \exp \left[-2 i \int_{0}^{z} \delta\left(z^{\prime}\right) \mathrm{d} z^{\prime}\right], \\
& \frac{\mathrm{d} \hat{a}_{2}}{\mathrm{~d} z}=i K^{*}(z) \hat{a}_{1} \exp \left[2 i \int_{0}^{z} \delta\left(z^{\prime}\right) \mathrm{d} z^{\prime}\right],
\end{aligned}
$$


where the local detuning parameter is defined as

$$
\begin{aligned}
\delta(z) & =\frac{1}{2}\left[\beta_{1}-\beta_{2}-\frac{2 \pi}{\Lambda(z)}+\Delta \beta_{1}(z)-\Delta \beta_{2}(z)\right] \\
& =\xi+\frac{1}{2}\left[\Delta \beta_{1}(z)-\Delta \beta_{2}(z)-2 \pi \frac{\mathrm{d} \nu}{\mathrm{d} z}\right]
\end{aligned}
$$

In the above expression, we also introduce the $z$-independent detuning parameter $\xi$, which is a function of the wavelength:

$$
\xi(\lambda)=\frac{1}{2}\left[\beta_{1}(\lambda)-\beta_{2}(\lambda)-\frac{2 \pi}{\bar{\Lambda}}\right] .
$$

The resonance condition implies that $\xi \cong 0$ for the wavelength range under consideration. We now make the following variable transformations:

$$
\begin{aligned}
& \nu_{1}(z, \xi)=\hat{a}_{1}(z) \exp (i \xi z), \\
& \nu_{2}(z, \xi)=\hat{a}_{2}(z) \exp (-i \xi z) .
\end{aligned}
$$

The transformed mode amplitudes satisfy the ZakharovShabat (ZS) equation:

$$
\begin{aligned}
& \mathrm{d} \nu_{1}(z, \xi) / \mathrm{d} z-i \xi \nu_{1}(z, \xi)=q(z) \nu_{2}(z, \xi), \\
& \mathrm{d} \nu_{2}(z, \xi) / \mathrm{d} z+i \xi \nu_{2}(z, \xi)=-q^{*}(z) \nu_{1}(z, \xi),
\end{aligned}
$$

where $q(z)$ is the complex coupling potential given by

$$
q(z)=i\left\{\kappa_{12} \sin [\mu(z) \pi] / \pi\right\} \exp [i \theta(z)]
$$

with the phase function

$$
\theta(z)=2 \pi \nu(z)-\left(\kappa_{11}-\kappa_{22}\right) \int_{0}^{z} \mu\left(z^{\prime}\right) \mathrm{d} z^{\prime} .
$$

Given the scattering coefficient, which is related to the filter response, the GLM inverse-scattering method can be used to derive the corresponding coupling potential $q(z){ }^{11}$ With the aid of Eqs. (21) and (22), the grating pattern can thus be derived as shown in Section 4 .

\section{TRANSFER-MATRIX METHOD FOR NONUNIFORM BINARY WAVEGUIDE GRATINGS}

We have previously developed a transfer-matrix method based on a mode-matching technique for the modeling of a photoinduced binary LPFG. ${ }^{7}$ In this approach, mode fields of region 1 are derived from those of region 0 by perturbation expansion to first order along with the corresponding propagation constants. The expansion coefficients to first order are successively expressed by the parameters used in coupled-mode theory. By requiring the continuities of the tangential electric and magnetic fields on the heterointerfaces of regions 0 and 1 , we can use a set of fundamental transfer matrices to describe the evolution of mode amplitudes as the wave field propagates through the LPFG. The details of the transfermatrix method for a binary LPFG can be found in Ref. 7 . In the following, we shall briefly review the fundamental transfer matrices and apply these matrices to the nonuniform binary LPFG shown in Fig. 1. Following the notation conventions in Ref. 7, we will henceforward use sym- bols with a bar above to represent quantities in region 0 , while the unbarred ones are for region 1 . Let the two mutually coupled-mode amplitudes be represented as a column vector $\mathbf{A}=\left(A_{1}, A_{2}\right)^{\mathrm{T}}$ [cf. Eq. (9)], where the superscript $\mathrm{T}$ indicate transpose. For the incidence from region 0 to region 1 , the changes in mode amplitudes can be expressed as $\mathbf{A}=\mathbf{F}^{(1 \mid 0)} \overline{\mathbf{A}}$ with the following interface matrix:

$$
\mathbf{F}^{(1 \mid 0)}=\left[\begin{array}{cc}
\alpha_{1} & \gamma \\
-\gamma^{*} & \alpha_{2}
\end{array}\right]
$$

And similarly, for incidence from region 1 to region 0 , we have $\overline{\mathbf{A}}=\mathbf{F}^{(0 \mid 1)} \mathbf{A}$ with

$$
\mathbf{F}^{(0 \mid 1)}=\left[\begin{array}{cc}
\alpha_{1} & -\gamma \\
\gamma^{*} & \alpha_{2}
\end{array}\right]
$$

where the off-diagonal element $\gamma$ represents the crosscoupling through the heterointerface and is given by

$$
\gamma=\frac{\kappa_{12}}{\beta_{1}-\beta_{2}} .
$$

$\alpha_{1}$ and $\alpha_{2}$ in Eqs. (23) and (24) are the self-transmission coefficients and are expressed by

$$
\alpha_{j}=1-D_{j} / 2 \quad(j=1,2),
$$

where the explicit form of $D_{j}$ can be found in Appendix A of Ref. 7. This term, $D_{j}$, accounts for the renormalization factor of the perturbed mode fields. In the ideal case of two-mode couplings, we shall assume that

$$
\alpha_{1}=\alpha_{2}=\sqrt{1-|\gamma|^{2}} \equiv \alpha .
$$

As for free propagation through region 1 , the mode amplitudes acquire a phase change and can be described by $\mathbf{A}(z)=\mathbf{P}^{(1)}(z) \mathbf{A}(0)$, where the phase matrix $\mathbf{P}^{(1)}$ is

$$
\mathbf{P}^{(1)}(z)=\left[\begin{array}{cc}
\exp \left[i\left(\beta_{1}+\kappa_{11}\right) z\right] & 0 \\
0 & \exp \left[i\left(\beta_{2}+\kappa_{22}\right) z\right]
\end{array}\right] .
$$

And similarly, we have the following for region $0: \quad \overline{\mathbf{A}}(z)$ $=\mathbf{P}^{(0)}(z) \overline{\mathbf{A}}(0)$ with

$$
\mathbf{P}^{(0)}(z)=\left[\begin{array}{cc}
\exp \left(i \beta_{1} z\right) & 0 \\
0 & \exp \left(i \beta_{2} z\right)
\end{array}\right] .
$$

Now we consider a unit period centered at $z=z_{n}$ as shown in Fig. 2. The local grating period corresponding to the $n$th region 1 is defined in the average sense:

$$
\Lambda_{n}=(1 / 2)\left[\left(z_{n+1}-z_{n}\right)+\left(z_{n}-z_{n-1}\right)\right] .
$$

The length of the right region 0 is

$$
l_{n}=(1 / 2)\left[\left(z_{n+1}-z_{n}\right)-w_{n}\right],
$$

while that of the left is

$$
l_{n}^{\prime}=(1 / 2)\left[\left(z_{n}-z_{n-1}\right)-w_{n}\right] .
$$

Note that, from the above definition, we have $w_{n}+l_{n}$ $+l_{n}^{\prime}=\Lambda_{n}$. The transfer matrix of the $n$th period is then given by 


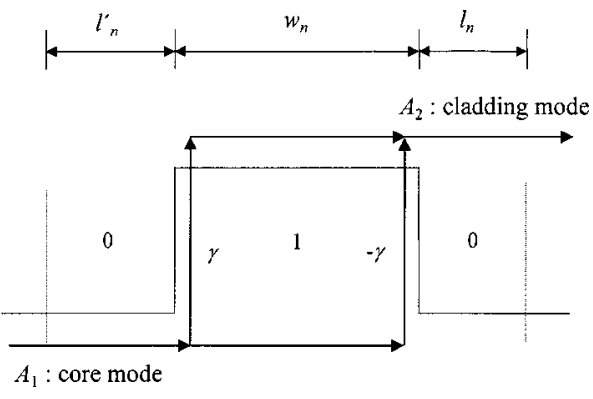

Fig. 2. Schematic diagram and corresponding parameters of a unit period. Also shown are couplings of the core mode to the phase-matched cladding mode through the two heterointerfaces.

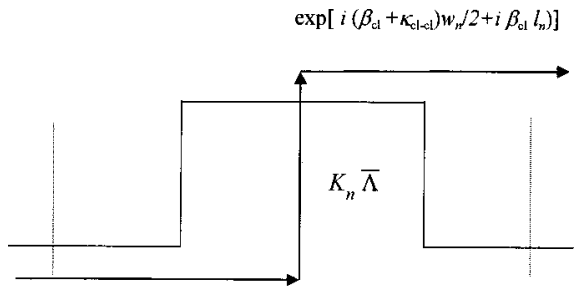

$\exp \left[i \beta_{\mathrm{sc}} l_{n}^{\prime}+i\left(\beta_{\mathrm{cc}}+\kappa_{\mathrm{cos}-\mathrm{c}}\right) w_{n} / 2\right]$

Fig. 3. Equivalent coupling to that in Fig. 2 from the core mode to the cladding mode; here $K_{n}$ is the equivalent coupling coefficient of the period.

$$
\begin{aligned}
\mathbf{F}_{n} & =\mathbf{P}^{(0)}\left(l_{n}\right) \mathbf{F}^{(0 \mid 1)} \mathbf{P}^{(1)}\left(w_{n}\right) \mathbf{F}^{(1 \mid 0)} \mathbf{P}^{(0)}\left(l_{n}^{\prime}\right) \\
& =\exp \left(i \theta_{n}\right)\left[\begin{array}{cc}
A_{n} & \Gamma_{n} \\
-\Gamma_{n}^{*} & A_{n}^{*}
\end{array}\right],
\end{aligned}
$$

where the matrix elements are

$$
\begin{aligned}
A_{n}= & \alpha^{2} \exp \left[i \frac{\left(\Delta_{n}+\bar{\Delta}_{n}\right)+\sigma \mu_{n}}{2}\right] \\
& +|\gamma|^{2} \exp \left[-i \frac{\left(\Delta_{n}-\bar{\Delta}_{n}\right)+\sigma \mu_{n}}{2}\right], \\
\Gamma_{n}= & \exp \left[i\left(\beta_{1}-\beta_{2}\right)\left(l_{n}-l_{n}^{\prime}\right)\right] \\
& \times(2 i \alpha \gamma) \sin \left(\frac{\Delta_{n}+\sigma \mu_{n}}{2}\right) .
\end{aligned}
$$

The following parameters are introduced:

$$
\theta_{n}=\bar{\beta} \Lambda_{n}+\left(\kappa_{11}+\kappa_{22}\right) w_{n} / 2
$$

is the global phase shift through the $n$th period, $\bar{\beta}=\left(\beta_{1}+\beta_{2}\right) / 2$ is the averaged propagation constant of the two modes, $\mu_{n}=w_{n} / \Lambda_{n}$ is the local duty ratio for the $n$th period, and

$$
\Delta_{n}=\left(\beta_{1}-\beta_{2}\right) w_{n}, \quad \bar{\Delta}_{n}=\left(\beta_{1}-\beta_{2}\right)\left(l_{n}+l_{n}^{\prime}\right)
$$

are the phase differences of the two mode amplitudes within regions 1 and 0 , respectively. We also define an important parameter characterizing the self-couplings:

$$
\sigma=\left(\kappa_{11}-\kappa_{22}\right) \bar{\Lambda} .
$$

We now consider the connection between the transfermatrix method and the coupled-mode theory by introducing an effective coupling coefficient. Note that the off-diagonal term of the transfer matrix $\mathbf{F}_{n}$ can be approximated in the continuous coupling sense as $\mathrm{d} A_{1} / \mathrm{d} z \approx\left[A_{1}(n+1)-A_{1}(n)\right] / \Lambda_{n} \approx \exp (i \bar{\theta}) \Gamma^{*} A_{2}(n) / \bar{\Lambda}$. Assuming that the grating parameters vary slowly enough that we may take $l_{n} \cong l_{n}^{\prime}$, we have

$$
\begin{aligned}
\exp \left(i \theta_{n}\right) \Gamma_{n}^{*}= & \exp \left[i \beta_{1} l_{n}^{\prime}+i\left(\beta_{1}+\kappa_{11}\right) \frac{w_{n}}{2}\right]\left(i \bar{\Lambda} K_{n}\right) \\
& \times \exp \left[i\left(\beta_{2}+\kappa_{22}\right) \frac{w_{n}}{2}+i \beta_{2} l_{n}\right] ;
\end{aligned}
$$

here we have introduced the following local effective coupling coefficient corresponding to $K(z)$ in Eq. (13) of the coupled-mode theory:

$$
K_{n}=\alpha \frac{\kappa_{12}}{\pi+\widetilde{\xi}} \sin \left[\mu_{n}(\pi+\sigma+\widetilde{\xi})\right],
$$

where $\widetilde{\xi}=\xi \bar{\Lambda}$ is the normalized detuning. Expression (39) can be interpreted as follows. The combined couplings of mode amplitudes from core to cladding through the two heterointerfaces of the $n$th period (see Fig. 2) can be represented as a single coupling taking place at $z=z_{n}$ with effective coupling $K_{n} \bar{\Lambda}$. This is also illustrated in Fig. 3. Note that when $\sigma \ll 1$ (small difference between self-coupling constants) and $\widetilde{\xi} \ll 1$ (narrow spectral range), expression (40) reduces to the result of coupled-mode theory [Eq. (13)]. However, the grating period of the LPFG is rather large; thus both $\sigma$ and $\widetilde{\xi}$ cannot be neglected completely. This is a special feature of the long periodicity. For a typical LPFG, the value of the self-coupling parameter is approximately $\sigma \cong \kappa_{01-01}^{\text {co-co }} \bar{\Lambda}$ $\cong 0.1$ to 1 , and $\xi_{\max } \bar{\Lambda} \cong 0.01$ to 0.1 ; thus the deviation from the coupled-mode theory is appreciable, and compensation should be made to optimize the design.

\section{FILTER DESIGN BASED ON THE INVERSE-SCATTERING METHOD WITH GENETIC ALGORITHM OPTIMIZATION}

We will design a binary LPFG with flatband spectral response in this section. The design is based on the GLM inverse-scattering method combined with a genetic algorithm to optimize the grating pattern. The details of the GLM method can be found in Ref. 11. To apply the GLM inverse-scattering method, we must specify the scattering coefficient of the ZS equation (20), and in the forwardcoupling case, one has to specify the following scattering coefficient $^{10,11}$ :

$$
r(\xi)=\nu_{2}(L, \xi) / \nu_{1}(L, \xi)
$$

where $L$ is the interaction length of the grating. Basically, the GLM equations are a set of coupled integral equations used to solve the unknown coupling potential $q(z)$ given the scattering coefficient $r(\xi)$. Song and Shin ${ }^{11}$ showed that the GLM integral equations can be transformed into a set of linear equations for $q(z)$ and thus can be exactly solved if $r(\xi)$ is a rational function of $\xi$. We will use this method in the following flatband LPFG filter design. The flatband filter is well approxi- 


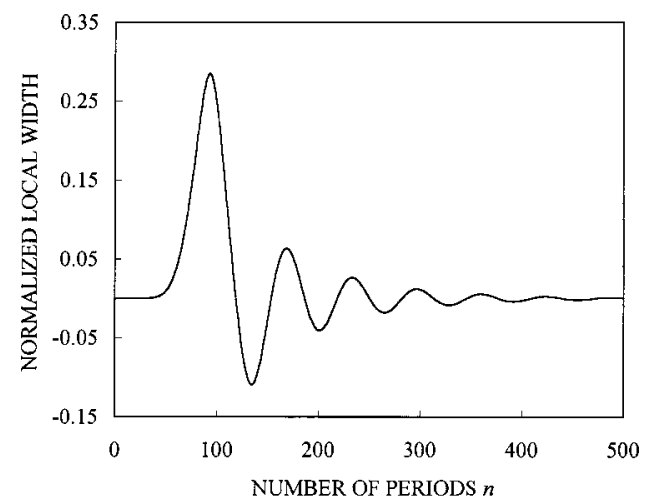

Fig. 4. Variation of the normalized local width $w_{n} / \bar{\Lambda}$ obtained from the GLM inverse-scattering method.

mated by a 13th-order Butterworth filter with maximum cross transmission being 0.5. The details of the design can be found in Refs. 10 and 16 . The reason for choosing a cross transmission of 0.5 is as follows. We want to construct an all-fiber MZ filter by cascading two LPFGs. ${ }^{17,18}$ The first LPFG splits light into two paths: One is within the core mode, while the other is through the propagation of the cladding mode. The second LPFG then combines light beams from these two paths. From the theory of MZ interferometry, the best visibility will occur when the power-splitting ratio is $0.5{ }^{17,18}$ Once the coupling potential is determined by the GLM method, the local duty ratio and period are obtained from Eqs. (21) and (22) as

$$
\begin{aligned}
& \mu(z)=\frac{1}{\pi} \sin ^{-1}\left[\pi \frac{|q(z)|}{\kappa_{12}}\right], \\
& \frac{1}{\Lambda(z)}=\frac{1}{\bar{\Lambda}}+\frac{1}{2 \pi} \frac{\mathrm{d} \theta}{\mathrm{d} z}+\frac{\sigma \mu(z)}{2 \pi \bar{\Lambda}} .
\end{aligned}
$$

From Eqs. (5) and (43), we can derive the source-number function as

$$
n(z)=\int_{0}^{z} \frac{\mathrm{d} z^{\prime}}{\Lambda\left(z^{\prime}\right)},
$$

whose inverse gives the positions of region 1 in each period. Then the corresponding parameters for the $n$th period are $\Lambda_{n}=\Lambda\left(z_{n}\right)$ and $w_{n}=\mu\left(z_{n}\right) \Lambda_{n}$ with $z_{n}=z(n)$. Figures 4 and 5 show the variation of normalized local width $w_{n} / \bar{\Lambda}$ and local period $\Lambda_{n} / \bar{\Lambda}$ with respect to the number of periods derived by the GLM inverse-scattering method. Note that a sign change of the grating width $w_{n}$ indicates the insertion of a $\pi$ phase shift at the crossing point. And this is implemented in the transfer-matrix model by multiplying by a matrix $\mathbf{P}^{(0)}(\bar{\Lambda} / 2)$ at the corresponding point.

In Fig. 6, we calculate the corresponding crosstransmission spectrum by using the transfer-matrix method. The cross transmission is defined as the relative power transfer from mode 1 to mode 2 at the exit end of the codirectional grating. It can be seen that the deviation from the desired result is appreciable. As discussed above, this is because the coupled-mode equation, as well as the ZS equation, is a continuous approximation to the discrete couplings of the binary LPFG. When we use the transfer-matrix model to calculate the filter spectrum of the grating pattern derived from the GLM method, there are significant discrepancies. This might be due to the smearing of the discrete couplings or to the effect of the self-couplings. Thus, based on the result from the GLM method, we use a genetic algorithm to compensate for these discrepancies and to optimize the grating design. Genetic algorithms have been successfully applied to the design of various optical filters, including FBG,${ }^{19}$ thin-film ${ }^{20}$ and rugate ${ }^{21}$ filters. A real-coded genetic algorithm ${ }^{20}$ is used in our optimization of the grating pattern. Let the grating parameters derived from the GLM method be $\left(w_{n}^{(\mathrm{GLM})}, \Lambda_{n}^{(\mathrm{GLM})}\right)$ for $n=1,2, \ldots, N$. We add a small correction to these parameters,

$$
w_{n}=w_{n}^{(\mathrm{GLM})}+\Delta w_{n}, \quad \Lambda_{n}=\Lambda_{n}^{(\mathrm{GLM})}+\Delta \Lambda_{n},
$$

and then use the genetic algorithm to optimize these corrections $\left(\Delta w_{n}, \Delta \Lambda_{n}\right)$. The genetic algorithm operates on a population of potential correction candidates. Each individual of the population can be represented by the following sequence:

$$
\mathbf{x}=\left\{\Delta w_{1}, \Delta \Lambda_{1} ; \Delta w_{2}, \Delta \Lambda_{2} ; \ldots, \Delta w_{n}, \Delta \Lambda_{N}\right\} .
$$

A suitable merit function used to return a single real number reflecting the total fitness of a specific correction is given $b y^{21}$

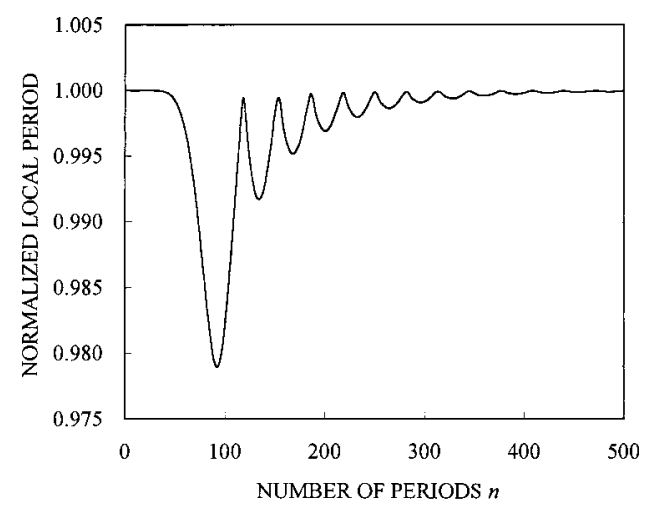

Fig. 5. Same as Fig. 4 but for the normalized local period $\Lambda_{n} / \bar{\Lambda}$.

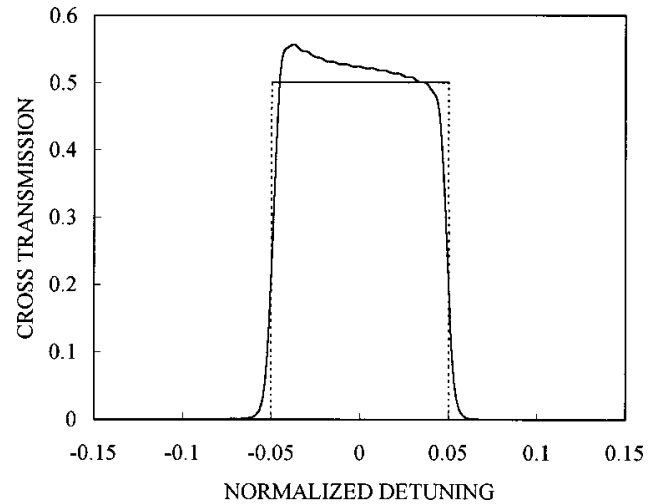

Fig. 6. Cross-transmission spectrum of the grating parameters in Figs. 4 and 5 calculated by the transfer-matrix model. The dotted lines represent the desired flatband spectrum. 


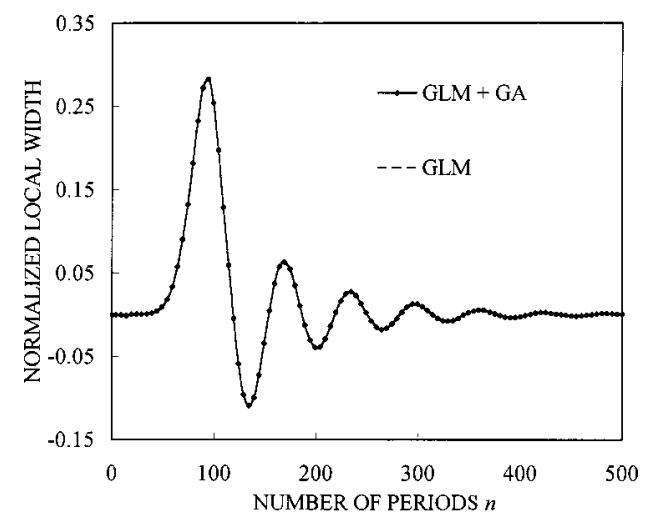

Fig. 7. Variation of the normalized local width $w_{n} / \bar{\Lambda}$ obtained from the GLM inverse-scattering method with genetic algorithm optimization. Also shown for comparison is the result obtained by using only the GLM method.

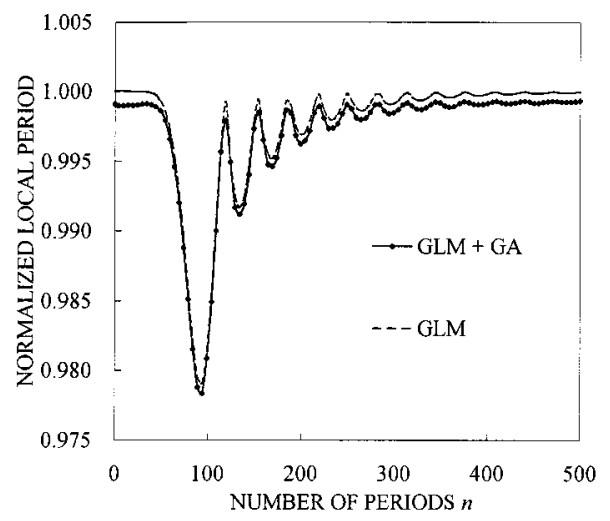

Fig. 8. Same as Fig. 7 but for the normalized local period $\Lambda_{n} / \bar{\Lambda}$.

$$
F(\mathbf{x})=\left\{\frac{1}{M} \sum_{j=1}^{M}\left[\frac{T\left(\lambda_{j} ; \mathbf{x}\right)-T_{\text {target }}\left(\lambda_{j}\right)}{\mathrm{d} T_{j}}\right]^{2}\right\}^{1 / 2},
$$

where $T\left(\lambda_{j} ; \mathbf{x}\right)$ is the cross transmission calculated by using the transfer-matrix method with grating parameters given by sequence $\mathbf{x}$ at wavelength $\lambda_{j}, T_{\text {target }}\left(\lambda_{j}\right)$ is the corresponding target cross transmission, and $\mathrm{d} T_{j}$ is the tolerance at $\lambda_{j}$. The genetic algorithm finds the optimum corrections $\mathbf{x}$ according to this figure of merit based on the rules of natural selections. Three primary operations are used to evolve the population of candidate corrections: selection, crossover, and mutation. The detailed definitions of these operations for real-coded genetic algorithms can be found, for example, in Ref. 20. The optimized grating parameters are shown in Figs. 7 and 8. For comparison, in these figures, we also plot the results obtained by using only the GLM method. It can be seen that although the corrections to the local width are small, corrections to the local period are quite appreciable. In Fig. 9, we calculate the corresponding crosstransmission spectrum by the transfer-matrix model; it can be seen that the filter response is improved by the genetic algorithm.

The above results are applied to the design of a binary LPFG with the following fiber parameters: core radius $a_{\mathrm{co}}=2.62 \mu \mathrm{m}$, cladding radius $a_{\mathrm{cl}}=62.5 \mu \mathrm{m}$, core index $n_{\text {co }}=1.4557$, and cladding index $n_{\mathrm{cl}}=1.45$. We choose the resonant cladding mode to be $\mathrm{LP}_{05}$. The reference period is determined by $\bar{\Lambda}=\lambda_{0} /\left(n_{01}^{\text {co }}-n_{05}^{\text {cl }}\right)=787.4 \mu \mathrm{m}$ for center wavelength $\lambda_{0}=1.55 \mu \mathrm{m}$. The calculated cross-coupling constant is $\kappa_{12}=\kappa_{01-05}^{\mathrm{co}-\mathrm{cl}}=0.266 \Delta n_{\mathrm{UV}}$. The self-coupling constant for the core mode is $\kappa_{01-05}^{\mathrm{co}-\mathrm{cl}}$ $=1.276 \Delta n_{\mathrm{UV}}$, while that for the cladding mode is taken to be zero. $^{2}$ The index modulation $\Delta n_{\mathrm{UV}}=1.576$ $\times 10^{-4}$. Figure 10 shows the transmission spectrum of the binary LPFG calculated by the transfer-method model taking into account the waveguide dispersion; it can be seen that a flatband transmission loss extends approximately from 1535 to $1565 \mathrm{~nm}$. It should be noted that the above analysis is basically applicable only to a given resonant cladding mode. This is because the difference between self-couplings $\kappa_{11}-\kappa_{22}$ is dependent on which cladding mode is being considered and is also related to the cross-coupling constant $\kappa_{12}$, which determines the desired transmission loss.

An all-fiber MZ filter with high fringe visibility can be constructed by symmetrically cascading two such LPFGs. ${ }^{17,18}$ The grating response is calculated as follows. Let $\mathbf{F}_{\mathrm{LPFG}}$ be the overall transfer matrix of a single flatband LPFG; then the transfer matrix corresponding to the MZ filter is

$$
\mathbf{F}_{\mathrm{MZ}}=\left(\mathbf{F}_{\mathrm{LPFG}}\right)^{\mathrm{T}} \mathbf{P}^{(0)}\left(L_{\mathrm{MZ}}\right) \mathbf{F}_{\mathrm{LPFG}},
$$

where the superscript $\mathrm{T}$ indicates transpose of the transfer matrix, which physically reverses the ordering of the

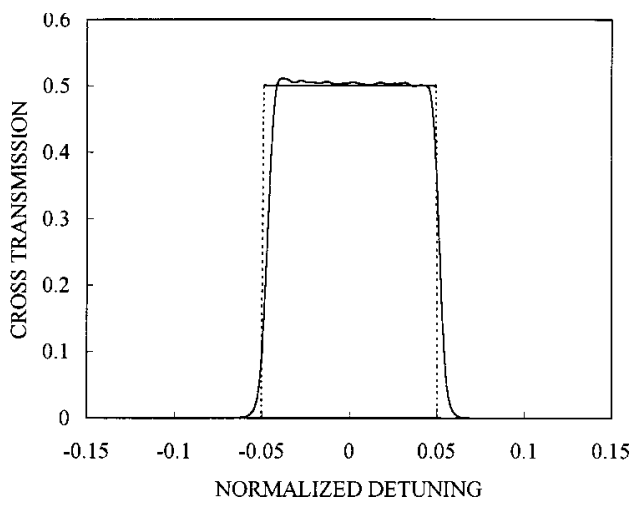

Fig. 9. Cross-transmission spectrum of the grating parameters in Figs. 7 and 8 optimized by genetic algorithm. The dotted lines represent the desired flatband spectrum.

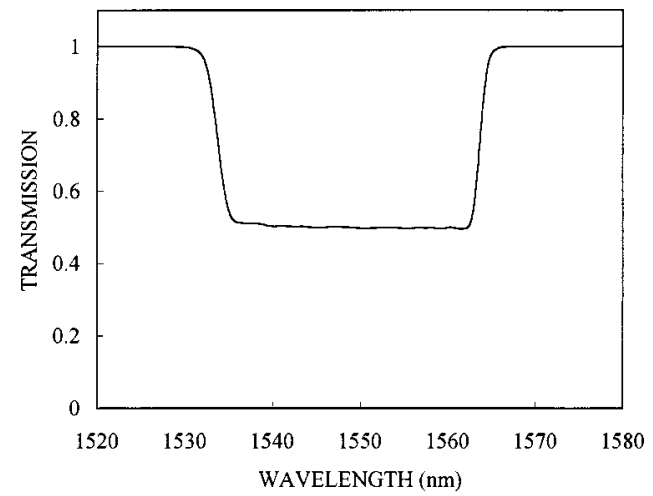

Fig. 10. Transmission spectrum of the synthesized flatband binary LPFG. 


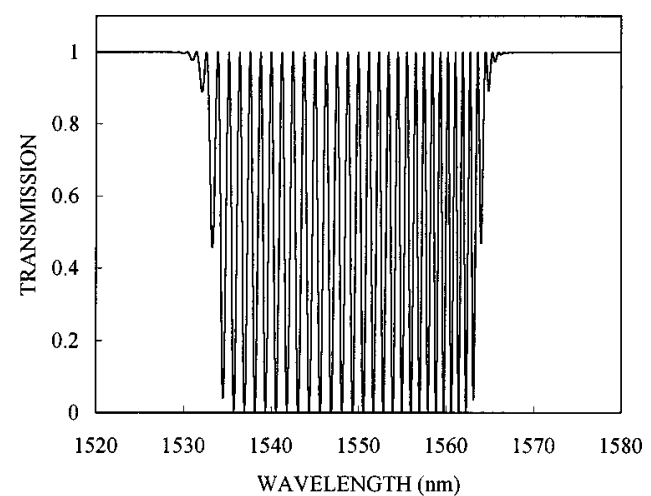

Fig. 11. Transmission spectrum of the all-fiber MZ filter composed of two symmetrically cascaded flatband LPFGs.

corresponding grating. The separation between the two gratings is chosen to be $L_{\mathrm{MZ}}=30 \bar{\Lambda}$. Figure 11 shows the calculated transmission spectrum in linear scale. A fringe pattern with high visibility over a spectral range of approximately $30 \mathrm{~nm}$ is obtained. Such a filter may have potential applications in wavelength-division multiplexing systems.

\section{CONCLUSION}

We have presented a procedure for the design of binary LPFG filters based on the GLM inverse-scattering method with genetic algorithm optimization. The coupling strength of the binary LPFG is varied with the local duty ratio. The index variation of the binary LPFG is expanded into a quasi-Fourier series by the Poisson sum formula. The zeroth-order expansion coefficient contributes to the self-couplings of the interacting modes, while the phase-matched first-order term determines the taper function of the coupling coefficient. A coupled-mode equation is presented for the application of the GLM inverse-scattering method. The grating response is calculated by using a transfer-matrix model developed previously by the authors. A connection between the transfer-matrix model and the coupled-mode theory is established for the nonuniform binary LPFG. It is found that the coupled-mode theory can serve only as an approximate modeling to the binary grating, since the discrete coupling nature of the binary LPFG is smeared out. From the transfer-matrix model, we derive a generalized local coupling coefficient, which under certain conditions can be reduced to the result obtained from the coupledmode theory. The grating parameters derived from the GLM inverse-scattering method are used as initial bases for the application of the genetic algorithm to optimize the grating design. We apply the above procedure to the design of a binary LPFG with flatband transmission spectrum and show that by symmetric cascading of two such gratings a high-visibility all-fiber MZ filter can be constructed.

\section{ACKNOWLEDGMENTS}

G. W. Chern is grateful to H. H. Liang for his help and useful discussions about the genetic algorithms. The authors are also grateful to the support in part by the Na- tional Science Council in Taiwan, Republic of China (R.O.C.), under contract NSC 90-2215E-002-007 and by the Education Ministry in Taiwan, R.O.C., under contract 89-E-FA06-2-4.

Address correspondence to Lon A. Wang at the location on the title page or by phone, 886-2-23635251; fax, 886-223656327; or e-mail, lon@ccms.ntu.edu.tw.

\section{REFERENCES}

1. A. M. Vengsarkar, P. J. Lemaire, J. B. Judkins, V. Bhatia, T. Erdogan, and J. E. Sipe, "Long-period fiber gratings as band-rejection filters," J. Lightwave Technol. 14, 58-65 (1996).

2. T. Erdogan, "Cladding-mode resonances in short- and longperiod fiber grating filters," J. Opt. Soc. Am. A 14, 17601773 (1997).

3. A. M. Vengsarkar, J. R. Pedrazzani, J. B. Judkins, P. J. Lemaire, N. S. Bergano, and C. R. Davidson, "Long-period fiber-grating-based gain equalizers," Opt. Lett. 21, 336-338 (1996).

4. J. R. Qian and H. F. Chen, "Gain flattening fibre filters using phase-shifted long period fibre gratings," Electron. Lett. 34, 1132-1133 (1998).

5. J. Bae, J. Chun, and S. B. Lee, "Equalization of the non-flat erbium gain spectrum using the multiport lattice filter model," in Optical Fiber Communications Conference, Vol. 2 of 2000 OSA Technical Digest Series (Optical Society of America, Washington, D.C., 2000), pp. 80-83.

6. W. H. Loh, M. J. Cole, M. N. Zervas, S. Barcelos, and R. L. Laming, "Complex grating structures with uniform phase masks based on the moving fiber-scanning beam technique," Opt. Lett. 20, 2051-2053 (1995).

7. G. W. Chern and L. A. Wang, "Transfer-matrix method based on perturbation expansion for periodic and quasiperiodic binary long-period gratings," J. Opt. Soc. Am. A 16, 2675-2689 (1999).

8. H. Sakata, "Sidelobe suppression in grating-assisted wavelength-selective couplers," Opt. Lett. 17, 463-465 (1992).

9. Y. H. Jan, G. A. Fish, L. A. Coldren, and S. P. DenBaars, "Demonstration of InP-InGaAsP vertical grating-assisted co-directional coupler filters and receivers with tapered coupling coefficient distributions," IEEE Photon. Technol. Lett. 9, 994-996 (1997)

10. G. W. Chern and L. A. Wang, "Analysis and design of almost-periodic vertical-grating-assisted codirectional coupler filters with nonuniform duty ratios," Appl. Opt. 39, 4629-4637 (2000).

11. G. H. Song and S. Y. Shin, "Design of corrugated waveguide filters by the Gel'fand-Levitan-Marchenko inversescattering method," J. Opt. Soc. Am. A 2, 1905-1915 (1985).

12. L. Poladian, "Simple grating synthesis algorithm," Opt. Lett. 25, 787-789 (2000).

13. R. Feced, M. N. Zervas, and M. A. Muriel, "An efficient inverse scattering algorithm for the design of nonuniform fiber Bragg gratings," IEEE J. Quantum Electron. 35, 11051115 (1999).

14. A. Ishimaru, "Theory of unequally spaced arrays," IRE Trans. Antennas Propag. 10, 691-702 (1963).

15. A. W. Snyder and J. D. Love, Optical Waveguide Theory (Chapman \& Hall, London, 1991).

16. K. A. Winick, "Design of grating-assisted waveguide couplers with weighted coupling," J. Lightwave Technol. 9, 1481-1492 (1991).

17. X. J. Gu, "Wavelength-division multiplexing isolation fiber filter and light source using cascaded long-period fiber gratings," Opt. Lett. 23, 509-510 (1998).

18. B. H. Lee and J. Nishii, "Dependence of fringe spacing on 
the grating separation in a long-period fiber grating pair," Appl. Opt. 38, 3450-3459 (1999).

19. J. Skaar and K. M. Risvik, "A genetic algorithm for the inverse problem in synthesis of fiber gratings," J. Lightwave Technol. 16, 1928-1932 (1998).

20. E. Michielssen, S. Ranjithan, and R. Mittra, "Optimal multilayer filter design using real coded genetic algorithm," IEE Proc. J Optoelectron. 139, 413-420 (1992).

21. P. L. Swart, A. P. Kotze, and B. M. Lacquet, "Effects of the nature of the starting population on the properties of rugate filters designed with the genetic algorithm," J. Lightwave Technol. 18, 853-859 (2000). 